(c) American Dairy Science Association, 2004.

\title{
Effect of Simultaneous Thawing of Multiple 0.5-mL Straws of Semen and Sequence of Insemination on Conception Rate in Dairy Cattle
}

\author{
J. C. Dalton, ${ }^{1, \star}$ A. Ahmadzadeh, ${ }^{1,}+$ B. Shafii, ${ }^{1,} \neq$ \\ W. J. Price, ${ }^{1,} \ddagger$ and J. M. DeJarnette ${ }^{2}$ \\ ${ }^{1}$ University of Idaho, Moscow 83843 \\ ${ }^{2}$ Select Sires, Inc. Plain City, OH, 43064
}

\section{ABSTRACT}

The objectives of this study were to determine 1) the effect of simultaneous thawing of multiple $0.5-\mathrm{mL}$ straws of semen and sequence of insemination (first, second, third, or fourth) on conception rates in dairy cattle, 2) whether the conception rates achieved following AI by professional AI (PAI) technicians and herdsman-inseminators (HI) differed, and 3 ) the effect of elapsed time from initiation of thawing straws of semen to seminal deposition on conception rates in dairy cattle. Four dairies with PAI and four with HI participated in the study. Initial data recorded included beginning thaw time, cow identification number, and time of seminal deposition. Herd records were retrieved following pregnancy diagnosis. Conception rates of dairy cows $(n=1025)$ were not affected by sequence of insemination (first, second, third, or fourth). Conception rates for herds using PAI were 40, 47, 41, and 50\%. Conception rates for herds using HI were 24, 20, 33, and $30 \%$. Average conception rates of dairy cows differed between PAI and HI (45 vs. $27 \%$, respectively). The difference in mean conception rate achieved by PAI and HI was not attributable to milk production, parity, service number or stage of lactation. The elapsed time from initial thaw to completion of fourth AI was shorter for PAI than for HI, 7.6 vs. 10.9 min, respectively. Although the average conception rate differed between PAI and HI, elapsed time from initial thaw to completion of fourth AI and sequence of insemination (first, second, third, or fourth) had no effect on conception rate within inseminator group.

(Key words: artificial insemination, multiple semen straws, elapsed time)

Abbreviation key: HI = herdsman-inseminator, PAI = professional AI.

Received October 31, 2003.

Accepted December 18, 2003.

Corresponding author: J. C. Dalton; e-mail: jdalton@uidaho.edu.

* $\uparrow$ Animal and Veterinary Science Department

$\ddagger$ Statistical Programs, College of Agricultural and Life Sciences

\section{INTRODUCTION}

As dairy producers manage larger herds, labor efficient management strategies such as once-daily $\mathrm{AI}$ and fixed-timed AI synchronization protocols are more common. Consequently, numerous cows must be inseminated on a given day. To facilitate AI in a timely manner, AI technicians routinely thaw multiple straws of semen simultaneously. Reports by Lee et al. (1997) and Goodell (2000) utilizing herdsman-inseminators (HI) suggest that thawing more than 2 straws at once results in reduced conception rates of the third and forth insemination in sequence. In contrast, Sprenger et al. (2001) and Kaproth et al. (2002) reported no differences in conception rates due to sequence of insemination (first through fourth) following multiple straw-thawing by professional AI (PAI) technicians. Furthermore, Brown et al. (1991) reported no difference in postthaw spermatozoal viability in vitro, as measured by percentage motility and percentage intact acrosomes, when up to 10 straws were thawed simultaneously.

In previous work (Lee et al., 1997; Goodell, 2000; Sprenger et al., 2001; Kaproth et al., 2002), the number of straws thawed simultaneously varied throughout the studies. Consequently, the interpretation of the number of straws that may be thawed simultaneously without effect on subsequent conception rate is difficult.

The objectives of this study were to determine 1) the effect of simultaneous thawing of multiple $0.5-\mathrm{mL}$ straws of semen and sequence of insemination (first, second, third, or fourth) on conception rates in dairy cattle, 2) whether conception rates achieved following AI by PAI technicians and HI differed, and 3) the effect of elapsed time from initiation of thawing straws of semen to seminal deposition on conception rates in dairy cattle.

\section{MATERIALS AND METHODS}

\section{Herds}

Between December 2000 and June 2001, 8 commercial herds located in Idaho, Washington, California, and 
Table 1. The effect of sequence of insemination on conception rates after simultaneous thawing of four 0.5$\mathrm{mL}$ straws of semen by professional AI (PAI) technicians and herdsman-inseminators (HI).

\begin{tabular}{llllll}
\hline & \multicolumn{4}{c}{ Conception rate, ${ }^{1} \%$ (pregnant/total) } \\
\cline { 2 - 5 } $\begin{array}{l}\text { Inseminator } \\
\text { category }\end{array}$ & 1st & Sequence of insemination & Mean \\
\cline { 2 - 5 } & $40(61 / 153)$ & $47(71 / 150)$ & $41(60 / 146)$ & $50(74 / 147)$ & $45^{\text {a }(266 / 596)}$ \\
PAI & $24(26 / 108)$ & $20(21 / 103)$ & $33(36 / 110)$ & $30(33 / 108)$ & $27^{\text {b }}(116 / 429)$ \\
\hline
\end{tabular}

${ }^{\mathrm{a}, \mathrm{b}}$ Mean conception rates between inseminator category are different $(P<0.01)$.

${ }^{1}$ Within inseminator category, no differences in conception rate were observed due to sequence of insemination.

Ohio participated in the study, 4 with PAI technicians and 4 with HI. Criteria for participation included usage of: a) once-daily AI, b) 0.5-mL straws of semen, and c) warm water thawing of straws of semen. Cooperating herds ranged in size from 800 to 3600 lactating cows. Average yearly milk yield ranged from $9545 \mathrm{~kg}$ per cow to $13,181 \mathrm{~kg}$ per cow. Cattle were housed in dry lots, covered free-stall housing, or a combination of dry lotcovered free-stall housing. Cattle were fed a TMR formulated to meet or exceed requirements for lactating cows (NRC, 2001).

\section{Detection of Estrus, Semen Handling, and Artificial Insemination}

In all herds, cows were restrained in headlocks once daily to facilitate tail chalk application, detection of estrus based on removed tail chalk, and AI. Semen used for AI was from 5 suppliers. Four 0.5-mL straws were required to be thawed simultaneously for data to be included in the study. The recommended semen handling protocol included thawing in a $35^{\circ} \mathrm{C}$ water bath for a minimum of $45 \mathrm{~s}$, removal of straws and assembly of AI syringes, and transport of 4 loaded AI syringes. Straws were provided thermal and hygienic protection during loading into AI syringes, transport, and insemination. The order in which cows received AI was random with respect to parity, number of services, and stage of lactation. The desired anatomical site of insemination was the body of the uterus. No retraining of HI or PAI technicians occurred before, during, or after the field study.

\section{Data Management}

Data recorded by the inseminator included herd name, date, AI technician name, beginning thaw time, time of seminal deposition, and cow identification number. All inseminators used a watch or mobile phone (with a clock) to monitor elapsed time from the beginning of thawing to seminal deposition. Following preg- nancy diagnoses by a veterinarian 38 to $45 \mathrm{~d}$ after insemination, farm records were retrieved from each cooperating herd. Individual cow information obtained included: 1) pregnancy status, 2) AI service sire, 3) DIM at time of AI, 4) milk yield on day of AI (or DHIA test day closest to AI), 5) lactation number, and 6) AI service number. Records were not included in the study if cows left the herd prior to pregnancy diagnosis, or cows were diagnosed pregnant but received 2 or more AI over 2 or more days, and (or) inseminator data sheets or cow records were incomplete.

\section{Statistical Analyses}

Chi-square analyses were used to test the effect of sequence of insemination and inseminator category on conception rate. The analysis of variance (ANOVA) procedure was used to compare conception rate, elapsed time, stage of lactation ( $\leq 95 \mathrm{~d}, 96$ to $120 \mathrm{~d}$, and $\geq 121$ d), milk yield, lactation number, and service number between the AI groups. All statistical analyses were carried out using SAS (SAS, 2000).

\section{RESULTS AND DISCUSSION}

Conception rates were not affected by sequence of insemination (first, second, third, or fourth) for PAI technicians or HI. Conception rates for herds using PAI technicians were $40,47,41$, and $50 \%$. Conception rates for herds with HI were 24, 20, 33, and 30\% (Table 1). With respect to PAI technicians, these results are similar to those of Sprenger et al. (2001) and Kaproth et al. (2002), in which sequence of insemination had no effect on conception rates. In contrast, these results disagree with those of Lee et al. (1997) and Goodell (2000) who reported decreased conception rates of the third and fourth insemination in sequence, when $\mathrm{HI}$ thawed more than two straws at once. A possible reason for the disagreement might be that Lee et al. (1997) and Goodell (2000) used a limited number of cattle in their studies (89 and 180, respectively). In addition, Goodell (2000) 
Table 2. Least squares means (LSM) for milk yield, parity, and service number for cattle inseminated by professional AI (PAI) technicians and herdsman-inseminators (HI).

\begin{tabular}{|c|c|c|c|c|c|c|c|}
\hline \multirow{2}{*}{$\begin{array}{l}\text { Inseminator } \\
\text { category }\end{array}$} & \multirow[b]{2}{*}{$\mathrm{n}^{1}$} & \multicolumn{2}{|c|}{ Milk (kg) } & \multicolumn{2}{|c|}{ Parity (no.) } & \multicolumn{2}{|c|}{ Service (no.) } \\
\hline & & LSM & $\mathrm{SE}$ & LSM & $\mathrm{SE}$ & LSM & $\mathrm{SE}$ \\
\hline PAI & 596 & 40.73 & 2.1 & $2.7^{\mathrm{a}}$ & 0.13 & $1.65^{\mathrm{a}}$ & 0.22 \\
\hline HI & 429 & 39.24 & 2.5 & $2.0^{\mathrm{b}}$ & 0.15 & $2.63^{\mathrm{b}}$ & 0.26 \\
\hline
\end{tabular}

used only 7 cows for the fourth insemination in sequence, which makes data interpretation difficult.

The lack of an effect of sequence of insemination on conception rates agrees with in vitro results of Brown et al. (1991). Using water baths, Brown et al. (1991) studied the effects of thawing groups of $2,5,10,15$, and 20 straws $(0.5 \mathrm{~mL})$ of semen on posthaw spermatozoal viability. Brown et al. (1991) reported no difference in percentage motility and percentage intact acrosomes immediately postthaw, when up to 10 straws were thawed simultaneously.

The mean conception rate achieved differed between PAI technicians and HI $(P<0.01 ; 45$ vs. $27 \%$, respectively) (Table 1). Cattle inseminated by PAI technicians were of increased parity $(P<0.05 ; 2.7 \pm 0.13$ vs. $2.0 \pm$ 0.15 lactations) and had a decreased number of services $(P<0.05 ; 1.65 \pm 0.22$ vs. $2.63 \pm 0.26)$, but did not differ in milk yield compared with cattle inseminated by HI (Table 2). Within inseminator classification, there were no effects of parity, service number, or stage of lactation (Table 3 ) on the conception rate achieved by PAI technicians or HI.

The misdiagnosis of estrus contributes to poor reproductive performance and has reduced the economic impact of AI (Senger, 1994). Using milk progesterone analyses, Reimers et al. (1985) reported the proportion of cows not in or near estrus when inseminated varied from 0 to $60 \%$ among dairy herds. In a study of 3 California dairy herds, the accuracy of tail chalk as an estrus detection device varied from 38 to $50 \%$, based on progesterone in milk or subsequent pregnancy after AI (Phatak and Touchberry, 1988). Taken together with the average conception rates achieved by $\mathrm{HI}$ and PAI technicians ( 27 vs. $45 \%$ ), a possible explanation for the conception rate difference between PAI technicians and HI may be related to estrus detection accuracy.

Only one study evaluating AI technique between PAI technicians and $\mathrm{HI}$ exists in the literature (Peters et al., 1984). Using radiography in a controlled laboratory setting, Peters et al. (1984) reported that there were no differences between PAI technicians and HI in AI syringe placement or distribution of the inseminate in various anatomical locations. It is not known whether $\mathrm{PAI}$ technicians and $\mathrm{HI}$ differed in $\mathrm{AI}$ technique in the present study conducted on commercial dairies.

Both PAI technicians and HI thawed straws of semen, assembled AI syringes, and inseminated cattle in a timely manner. The elapsed time from initial thaw to completion of the first AI was not different between PAI technicians and $\mathrm{HI}(4.2 \pm 0.17$ vs. $5.8 \pm 0.22 \mathrm{~min}$, respectively). The elapsed time from initial thaw to completion of fourth AI was shorter for PAI technicians than for HI $(7.6 \pm 0.22$ vs. $10.9 \pm 0.38 \mathrm{~min} ; P<0.01)$. Nevertheless, the lower conception rate observed following AI by HI was not likely due to an extended time factor. When $0.5-\mathrm{mL}$ semen straws were held at a constant temperature after thawing, Kaproth et al. (2002) reported no difference in mean progressive spermatozoal motility at 5 and 20 min postthaw. However, Kaproth et al. (2002) also reported a decrease in mean progressive spermatozoal motility from 5 to $20 \mathrm{~min}$ postthaw when $0.5-\mathrm{mL}$ semen straws were thawed at $35^{\circ} \mathrm{C}$ but held at $22^{\circ} \mathrm{C}$. It is possible that failure to maintain straws at a constant temperature during $\mathrm{AI}$

Table 3. Influence of stage of lactation on mean conception rates achieved by professional AI (PAI) technicians and herdsman-inseminators (HI). ${ }^{1}$

\begin{tabular}{lllll}
\hline & \multicolumn{3}{c}{ Conception rate } \\
\cline { 2 - 5 } & \multicolumn{2}{c}{ PAI } & & HI \\
\cline { 2 - 5 } Stage of lactation & LSM & SE & LSM & SE \\
\hline$\leq 95$ d & 0.46 & 0.03 & 0.30 & 0.06 \\
96 to $120 \mathrm{~d}$ & 0.40 & 0.07 & 0.25 & 0.10 \\
$\geq 121 \mathrm{~d}$ & 0.42 & 0.08 & 0.25 & 0.05 \\
\hline
\end{tabular}

${ }^{1}$ Within PAI technicians and HI, there was no influence of stage of lactation on mean conception rate. 
syringe assembly and transport to the cow is one of many contributing factors to the decreased conception rate achieved by $\mathrm{HI}$.

The results presented support the suggestion of Kaproth et al. (2002) that careful inseminators may thaw up to 4 straws simultaneously without a decline in conception rate provided inseminations are completed in a timely manner. Nevertheless, a general recommendation as to the number of straws that may be thawed simultaneously detracts from the overall importance of proper semen handling for successful AI. Conception rates are most likely to be maximized when PAI technicians and HI a) accurately identify cows in estrus, b) follow AI stud's recommendations for thawing semen, c) prevent direct straw-to-straw contact during thawing to avoid decreased postthaw spermatozoal viability as a result of straws freezing together (Brown et al., 1991), d) utilize appropriate hygienic procedures, e) maintain thermal protection of straws during AI syringe assembly and transport to the cow, and f) deposit semen in the uterus of the cow within approximately $15 \mathrm{~min}$ after thawing.

\section{CONCLUSIONS}

Although the average conception rate differed between PAI technicians and HI, elapsed time from initial thaw to completion of fourth $\mathrm{AI}$ and sequence of insemination (first, second, third, or fourth) had no effect on conception rate within inseminator group. Therefore, simultaneous thawing of four $0.5-\mathrm{mL}$ straws of semen does not impact conception rates, provided the inseminators strictly adhere to recommended semen handling procedures coupled with accurate identification of cows in estrus, and with deposition of semen completed within 15 min after thawing. This study should not be interpreted as a general recommendation to thaw 4 straws simultaneously. Inseminators that fail to accurately identify cows in estrus and (or) fail to properly handle semen will achieve unacceptable conception rates regardless of the number of straws thawed. Fu- ture efforts to improve the results of AI should focus on increasing and maintaining the insemination skills of all inseminators as well as improving the accuracy of estrus detection.

\section{ACKNOWLEDGMENTS}

This research was supported by a grant to Joseph C. Dalton and Amin Ahmadzadeh from Select Sires Inc., Plain City, OH. The authors thank Ken Rowley and Larry Lowe (All West/Select Sires), T. J. Lawrence (Cache Valley/Select Sires), Scott Braden (COBA/Select Sires), Tom Kasper, Reagon Hatch, George Phillips, and Steve Marquardt for their cooperation.

\section{REFERENCES}

Brown, D. W., P. L. Senger, and W. C. Becker. 1991. Effect of group thawing on post-thaw viability of bovine spermatozoa packaged in .5 milliliter French straws. J. Anim. Sci. 69:2303-2309.

Goodell, G. 2000. Comparison of AI pregnancy rates in dairy cattle by order of preparation of insemination straws. J. Anim. Sci. 78:(Suppl. 1):229. (Abstr.)

Kaproth, M. T., J. E. Parks, G. C. Grambo, H. E. Rycroft, and J. A. Hertl. 2002. Effect of preparing and loading multiple insemination guns on conception rate in two large commercial dairy herds. Theriogenology 57:909-921.

Lee, C. N., T. Z. Huang, and A. B. Sagayaga. 1997. Conception rates in dairy cattle are affected by the number of semen straws thawed for breeding. J. Dairy Sci. 80:(Suppl. 1):151. (Abstr.)

National Research Council. 2001. Nutrient Requirements of Dairy Cattle. 7th rev. ed. Natl. Acad. Sci., Washington, DC.

Peters, J. L., P. L. Senger, J. L. Rosenberger, and M. L. O'Connor. 1984. Radiographic evaluation of bovine artificial inseminating technique among professional and herdsman-inseminators using 0.5- and 0.25-ml French straws. J. Anim. Sci. 59:1671-1683.

Phatak, A., and R. W. Touchberry. 1988. Comparison of heat detection aids. J. Dairy Sci. 71:(Suppl. 1):136. (Abstr.)

Reimers, T. J., R. D. Smith, and S. K. Newman. 1985. Management factors affecting reproductive performance of dairy cows in the northeast United States. J. Dairy Sci. 68:963-977.

SAS Inst., Inc. 2000. SAS Online Doc, version 8. SAS Inst., Inc. Cary, NC.

Senger, P. L. 1994. The estrus detection problem: New concepts, technologies, and possibilities. J. Dairy Sci. 77:2745-2753.

Sprenger, M. J., J. M. DeJarnette, and C. E. Marshall. 2001. Conception rates of sequential inseminations after batch-thawing multiple straws of semen: A professional technician case study. J. Anim. Sci. 79:(Suppl. 1):253. (Abstr.) 